\title{
Revisión sobre la Utilización de Servicios de Salud, 1996-2006
}

\section{A review of health-service use (1996-2006)}

\author{
Myriam Ruiz-Rodríguez ${ }^{1}$ y Rosario Valdez-Santiago ${ }^{2}$ \\ 1 Departamento de Salud Pública, Universidad Industrial de Santander (UIS). Bucaramanga, Colombia. \\ myriam@uis.edu.co \\ 2 Centro de Investigación en Sistemas de Salud, Instituto Nacional de Salud Pública de México (INSP) \\ Cuernavaca, México. rvaldez@insp.mx
}

Recibido 16 Julio 2007/Enviado para Modificación 23 Enero 2008/Aceptado 28 Marzo 2008

\section{RESUMEN}

Objetivo Revisar, en artículos publicados durante el período 1996-2006, cuáles son los supuestos ontológicos, metodológicos y epistemológicos prevalentes en los estudios de la utilización de servicios de salud.

Material y métodos Para asegurar la identificación de estudios relevantes se realizó la búsqueda de los artículos indexados en la base de datos PubMed.

Resultados Un total de 34 artículos fueron incluidos en el análisis final. El 70,6 \% fueron realizados en Estados Unidos de América, 30 \% trató con población en desventaja social y el objeto de análisis más frecuentemente estudiado fue la visita médica (50\%). Treinta y un estudios (91,2\%) abordaron el problema de estudio desde la aproximación cuantitativa y tres desde la cualitativa. Las variables más estudiadas desde la perspectiva cuantitativa fueron las del individuo en 90,4\% de los estudios, seguidos de las variables del contexto $(45,2 \%)$ y del sistema de salud $(41,9 \%)$.Los análisis estadísticos de los estudios de corte cuantitativo se realizaron utilizando modelos de regresión múltiple y sólo 19,4 \% de los análisis incorporaron modelos que captan la complejidad del fenómeno de la utilización. Análisis del discurso y de contenido fueron realizados en los estudios cualitativos.

Conclusiones Los hallazgos reflejan el carácter reducible del fenómeno de la utilización a términos objetivos y necesariamente tiende a oscurecer que la utilización es una es una practica social integradora. Se requiere una perspectiva más amplia para abordar el fenómeno de la utilización. El uso de aproximaciones cuantitativas y cualitativas nos arroja resultados distintos que ayudan a configurar una visión integral de una misma problemática.

Palabras Clave: Servicios de salud, accesibilidad, utilización (fuente: DeCS, BIREME).

\footnotetext{
ABSTRACT

Objective Reviewing research papers published during 1996-2006 regarding ontological, methodological and epistemological proposals regarding the use of health services.
} 
Material and Methods Pub Med was screened for identifying the most relevant research carried out to date in the field. The key words <health services $><$ accessibility> were used.

Results 34 papers were included in the final analysis. $70,6 \%$ referred to health service use in the USA; $30 \%$ analysed the socially disadvantaged population, focusing mainly on visits to the doctor (50 \%). 31 papers $(91,2 \%)$ used a quantitative approach; the most studied variables in this approach regarded the individual $(90,4 \%)$ the context (45,2\%) and the health system (41,9\%). The quantitative approaches used multiple regression for their statistical analysis; only $19,4 \%$ of them incorporated more complex statistical models for analysing health service use. Qualitative studies analysed both discourse and content.

Conclusions The findings reflected how the nature of health service use becomes reduced to using objective terms which necessarily tend to hide that such use is dealing with social integration. A wider perspective is needed for tackling health service use. Complementary (qualitative-quantitative) approaches provide different results helping to shape an integral vision of the same problem.

Key Words: Health service, use, accessibility (source: MeSH, NLM).

A pesar de la existencia de abundante literatura sobre el tema de la utilización de servicios de salud, y los importantes avances que se han alcanzado en el desarrollo de modelos teóricos y métodos de análisis, existen pocos trabajos que aborden mediante revisión de artículos publicados cuáles son las perspectivas investigativas teóricas-conceputales prevalentes en el estudio de la utilización. En cambio, es más frecuente encontrar revisiones de temas que se orientan a identificar los marcos conceptuales más utilizados para abordar el estudio de la utilización, y las revisiones de estudios empíricos sobre los factores más importantes que influyen en ella.

Mendoza-Sassi y Béria (1) hicieron una revisión sistemática entre los años 1970 y 1999 cuyo objetivo fue identificar cuáles eran los factores más importantes y consistentes que afectaban la utilización de servicios de salud, cuál es el efecto que tienen y cuáles pueden ser considerados barreras o, por el contrario, facilitadores del acceso. Ellos encontraron que entre los factores demográficos, la población infantil y anciana, así como las mujeres en edad fértil utilizan más los servicios. Entre los socioeconómicos, la clase social con menos recursos económicos y el grupo con menos educación se asociaron con la utilización, y que el mayor uso está mediado por una mayor necesidad en salud.

Travassos y Martins (2) realizaron una revisión de los conceptos de acceso y utilización de servicios de salud con el propósito de analizar cómo estos 
conceptos se interrelacionan entre sí. Estas autoras destacan cómo el acceso es un concepto complejo que cambia a lo largo del tiempo de acuerdo con el contexto, y la visión predominante es que el acceso se relaciona con factores de la oferta. De otro lado, estas autoras señalan que el uso de servicios de salud representa el centro del funcionamiento del sistema de salud y es una expresión del acceso, pero que no está justificado solamente por éste, puesto que en el uso intervienen factores individuales y contextuales.

El objetivo de este manuscrito es revisar, en artículos publicados durante el período 1996-2006, cuáles son los supuestos ontológicos, metodológicos y epistemológicos prevalentes en los estudios de la utilización, además de identificar si las aproximaciones investigativas identificadas permiten visualizar la utilización de servicios de salud desde una perspectiva integral $(3,4)$. Así, la utilización de servicios de salud se visualiza como un proceso social, dinámico y complejo que pone en contacto a los actores, la población y el personal de salud, con el propósito de satisfacer una condición de salud determinada (4). En este sentido, se considera que la utilización asume diferentes patrones o perfiles según el contexto institucional y social en que se desenvuelven, tanto población como sistema de salud, e implica una interacción entre población y actores institucionales en un marco de realidades objetivas y subjetivas, individuales e institucionales necesarias para el logro de objetivos comunes: el mantenimiento o la recuperación de la salud.

Considerar a la utilización de servicios de salud desde una perspectiva amplia significa reconocer que en ella cconvergen no sólo la necesidad de atención (en términos de enfermedad), sino las experiencias pre-existentes de la población, sus creencias y aspectos culturales pero, sobre todo, el complejo contexto en que se da la prestación de servicios de salud y las condiciones con que cuenta la población en cuanto a activos personales, familiares y comunitarios.

\section{MATERIALES Y MÉTODOS}

Se hizo una revisión sistemática de artículos publicados bajo los siguientes criterios de selección: artículos originales en inglés, español y portugués, que incluyeran a personas de todas las edades, y que el evento a estudiar fuera la utilización de servicios de salud ambulatorios. Los estudios que consideraron la utilización de servicios de nivel ambulatorio no relacionada directamente con programas médicos fueron excluidos. Finalmente, también se excluyeron del análisis todos los artículos a los que no fue posible acceder al texto completo o 
que en su texto no especificaran cuáles fueron las variables independientes y dependientes.

La búsqueda de artículos fue realizada en la base de datos electrónica PubMed entre 1996 y 2006 usando los descriptores health services accessibility. Se creó un banco de datos en el programa Excel donde se ingresaron los artículos seleccionados. Los estudios se clasificaron de acuerdo con los objetivos, tipo de diseño, tipo de variables/categorías y tipo de análisis, de tal manera que nos permitiera identificar los supuestos ontológico, epistemológico y metodológicos $(5,6)$.

El fundamento ontológico $(6,7)$ hace referencia a cuál es la naturaleza de la realidad que se va a estudiar, y qué se puede conocer de ella. Así, desde la perspectiva cuantitativa se supone la existencia de un mundo real, por lo tanto, la naturaleza de la realidad es objetiva; se puede medir, contar; se puede aprehender. Por el contrario, desde la perspectiva cualitativa, la realidad es construida por los individuos, es vivida por los sujetos; las personas le atribuyen significados subjetivos a los eventos y actúan de acuerdo con estas interpretaciones.

El supuesto metodológico $(6,7)$ hace referencia a la naturaleza de las herramientas o arreglos que el investigador necesita desarrollar para poder conocer el objeto de estudio. En la investigación cuantitativa la estadística y sus procedimientos son la herramienta básica del análisis de los datos. En la cualitativa, sólo se puede acceder a la realidad comprendiendo el punto de vista de las personas que vivencian una experiencia dada, se utilizan procedimientos interpretativos que permitan el acercamiento a la subjetividad y a los significados y perspectivas de los participantes.

Como consecuencia de los dos supuestos anteriores, la naturaleza de la relación entre el investigador y el objeto de estudio (supuesto epistemológico) también difiere en ambas perspectivas (6,7). En la cuantitativa, la postura de quien conoce debe ser de distanciamiento, es decir, libre de valores, sin dejarse influenciar por él, ni influenciarlo. Por el contrario, en la cualitativa, el investigador es un co-partícipe en el descubrimiento y comprensión, y a la vez, es un instrumento, pues recaba su investigación en un escenario natural, por lo que no está libre de sesgos. 


\section{RESULTADOS}

Después de considerar los criterios de inclusión y exclusión, 34 artículos se incluyeron para su análisis. En la Tabla 1 se presentan las características más importantes de los estudios. La mayor proporción se realizaron en los Estados Unidos de América (EUA) y en población en desventaja social. La unidad de análisis más frecuente fue la visita a los servicios con (de las cuales la mayor proporción correspondió a visitas al médico), seguidas de uso de fuente regular de atención.

Tabla 1. Características de los estudios

\begin{tabular}{lrrl}
\hline Característica & No. & $\%$ & Referencias \\
\hline País del estudio & 24 & 70,6 & $8-29,39,41$ \\
EUA & 3 & 8,8 & $34-36$ \\
América Latina & 4 & 11,8 & $30-33$ \\
Europa & 2 & 5,9 & 37,38 \\
Asia y África & 1 & 2,9 & 40 \\
EUA-México & & & \\
Población estudiada & 10 & 29,5 & $8,12,19,20,23,24,46,27,39,41$ \\
Grupos en desventaja social & 7 & 20,6 & $13,18,30,32,34,35,38$ \\
Adultos mayores & 6 & 17,6 & $10,14,15,17,37,40$ \\
Población general >18 años de edad & 2 & 5,9 & 11,16 \\
Usuarios de seguro & 3 & 8,8 & $31,33,36$ \\
Grupos con enfermedades especificas & 6 & 17,6 & $9,21,22,25,28,29$ \\
Otros & & & \\
Unidad de análisis & 17 & 50,0 & $8,9,17-19,26-28,30-32,34-37,40,41$ \\
Visitas & 7 & 20,6 & $10,12,13,15,20,22,29$ \\
Uso de fuente regular de atención & 5 & 14,7 & $11,14,21,23,24$ \\
Visitas y barreras de uso & 3 & 8,8 & $33,38,39$ \\
Experiencia de uso & 2 & 5,9 & 16,25 \\
Otras & & & \\
\hline
\end{tabular}

Los estudios dilucidaron cuestiones como: los factores que afectan el acceso/utilización de servicios de salud (10,14-16,19,21,22,25,27,30,41); las diferencias, desigualdades e inequidades en la utilización de servicios de salud (8,12,17,18,31,32,34,35,37); los roles del seguro en la utilización (9,20,26,28,29); las barreras para el acceso/utilización $(11,13,23,24,40)$; las vivencias y expectativas sobre la utilización $(33,38,39)$ y los patrones de uso $(36)$.

Respecto a la aproximación al objeto de estudio hubo tres estudios cualitativos $(33,38,39)$ y 31 con aproximación cuantitativa. Todos los estudios cuantitativos fueron estudios de tipo observacional (cross sectional) y los cualitativos utilizaron la técnica de grupos focales. Es importante destacar que sólo en un estudio se tuvo en cuenta la perspectiva del proveedor y del usuario (33). En los artículos cuantitativos el método de análisis más utilizado fue el de regresión múltiple (80,6 \%). Los demás estudios usaron análisis complejos, como 
el análisis jerárquico $(10,14,21)$ análisis de endogeneidad $(9,28)$ y otro con método de descomposición de la disparidad entre grupos(29). En los estudios de tipo cualitativo dos de ellos enunciaron el método de análisis: análisis de discurso (33) y análisis de contenido (39).

Variables explicativas/categorías de análisis

En los estudios cuantitativos ( $\mathrm{n}=31$ ) se identificaron tres grupos de variables: las del individuo, las del sistema de salud y las del contexto. En la Figura 1 puede observarse que $41,9 \%(n=13)$ de estos estudios incluyeron variables que correspondían exclusivamente a uno de dichos grupos, siendo las de mayor frecuencia las variables del individuo $(n=9)$. Por el contrario, 17,9 \% $(11,14,19,26,29)$ incluyó variables de los tres grupos arriba mencionados.

Figura 1.Tipo de Variables explicativas del objeto de estudio

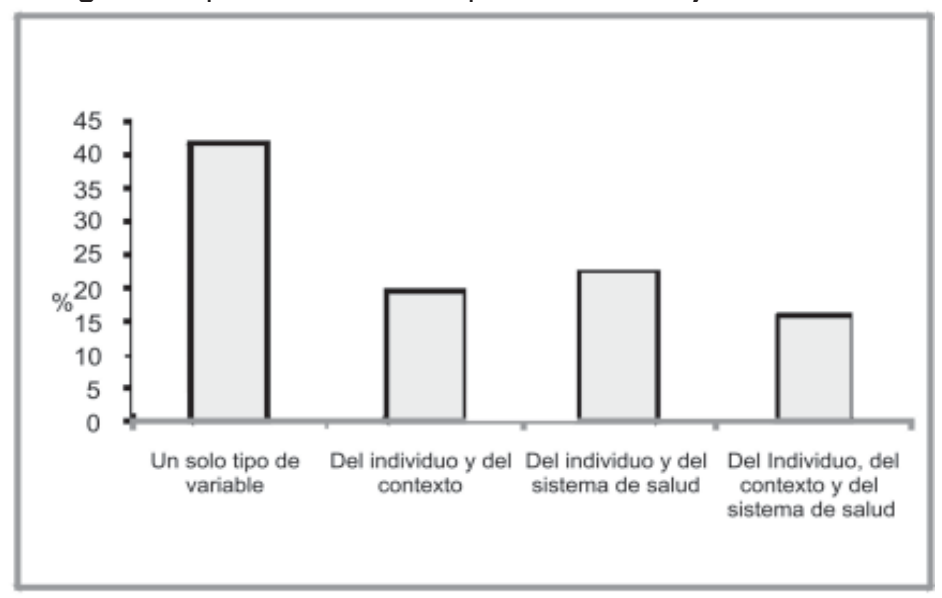

El sexo, tenencia de seguro, educación y estado de salud estaban presentes en todos los estudios que consideraron variables del individuo y en menor proporción otros atributos como etnicidad, raza, estatus marital, ingreso, lugar de residencia y contar con una fuente regular de atención. Las variables del sistema de salud fueron medidas en $41,9 \%$ de los estudios cuantitativos (8,10,11,13,14,16,19,23-26,29,37), encontrándose que los factores más estudiados fueron: disponibilidad de recurso humanos y físicos, tipo de servicios, tiempos de espera para obtener una cita, tiempo de espera en el consultorio y políticas institucionales, respectivamente.

En el tercer grupo se ubican las variables de contexto, definidas como aquellos factores del ambiente que reflejan el clima familiar, económico, la 
riqueza, políticas, demanda y dinámica de mercados, nivel de estrés y violencia, y las normas de la sociedad. El 45,1 \% incluyó factores de esta naturaleza $(9,11,12,14,15,17,19,21,22,26,27,29,31,34)$. El entorno familiar fue el más estudiado (ingreso familiar, estructura familiar, recursos económicos, educación) seguido del económico (tasa de desempleo, ingreso, ingreso per capita, desigualdad en el ingreso, entre otras), el cual fue estudiado en 4 de los 11 estudios que abordaron estas variables. Le siguen en su orden, las condiciones de la población (tasas de pobreza, estrato socioeconómico, violencia), el entorno demográfico (densidad poblacional), y contexto de demanda y mercado.

En los estudios cualitativos las categorías identificadas se derivan directamente de los análisis de los relatos de los participantes. Así, por ejemplo, Lester (33), identifica como una de sus categorías de análisis a las tensiones entre las perspectivas de los pacientes y médicos, sobre lo que es la enfermedad mental y su tratamiento.

\section{DISCUSIÓN}

El propósito de esta revisión fue identificar, a partir de los supuestos, ontológicos, metodológicos y epistemológicos, la aproximación investigativa prevalente en los estudios sobre utilización de servicios y analizar si ésta permite visualizar a la utilización desde una perspectiva integral. Solamente se consideraron investigaciones que fueron reportadas en revistas indexadas en PubMed. Ésta, por lo tanto, no es una revisión exhaustiva porque otras revistas científicas no indexadas en otras bases de datos pueden también tratar este tema. También reconocemos que no fueron incluidas revisiones de fuentes gubernamentales y no gubernamentales, ni tampoco tesis doctorales y de maestría. Sin embargo, consideramos que esta base de datos es una de las que contiene el mayor número de revistas con revisión de pares y, además, es una de las principales fuentes de comunicación científica para los profesionales de la salud y, por lo tanto, los artículos allí publicados reflejan las temáticas de estudio prevalentes en el estudio de la utilización de servicios de salud.

El paradigma dominante para abordar la problemática de utilización de servicios de salud es el cuantitativo, hecho que se constata al observar el tipo de diseño, variables y análisis dominantes. Los estudios revisados fallaron en reflejar la naturaleza multidimensional y compleja de la utilización, y el carácter interactivo del proceso de la utilización. Dieron muy poca cuenta de la forma como se relacionan los actores que participan en el sistema de prestación de servicios, en especial de aquellos sistemas que son complejos dada la multipli- 
cidad de actores que participan en la entrega de los servicios. Las variables estudiadas fueron en su gran mayoría de estructura del sistema de atención y de las características individuales. Sólo tres estudios evaluaron el punto de vista de los participantes respecto a la utilización de servicios.

Estos hallazgos reflejan el carácter reducible del fenómeno de la utilización a términos objetivos, y necesariamente tiende a invisibilizar que la utilización es una es una practica social integradora, que resulta de la construcción valorativa efectuada siempre en una cultura, en una sociedad y en una época determinada. En esta interacción convergen no sólo aspectos objetivos, tales como las características demográficas y económicas del paciente, el tipo de enfermedad, los recursos, la tecnología, sino aspectos subjetivos, como son el padecimiento, la cultura del paciente, las relaciones entre actores, la información, la confianza, las normas, los valores y las formas de organización de los sistemas de salud. Estos aspectos subjetivos son difícilmente medibles bajo el paradigma cuantitativo.

Estos resultados también reflejan, de alguna manera, que el estudio del fenómeno de la utilización de servicios de salud no es ajeno a la dicotomía que tradicionalmente se ha presentado entre la exigencia de objetividad de la ciencia positivista y las valoraciones y consideraciones relativas a la salud pública, lo cual ha sido el obstáculo para que se de cuenta de la complejidad del proceso salud-enfermedad-atención $(42,43)$. Esta situación se ha reflejado, entre otros aspectos, en lo que Granda (44) denomina salud pública convencional, la cual mira al proceso salud-enfermedad-atención como un objeto, y no como sujeto de acción, a ser intervenido por parte de la norma funcional y de la ciencia positivista.

Se requiere una perspectiva más amplia de abordaje del fenómeno de la utilización para lograr una mayor comprensión de este fenómeno y para que los resultados de las investigaciones sean más útiles para los tomadores de decisiones (45). Trascender los estudios de utilización con énfasis en el individuo y en la estructura del sistema de salud y, consecuentemente con ello la dominancia de metodologías positivistas, para transitar a una visión más holística que nos sugiera un estudio de la utilización más amplio, el cual refleje el carácter interactivo y multidimensional de la misma.

Para superar el reduccionismo del enfoque convencional es necesario propugnar por el uso de aproximaciones integradoras (46-49), con metodologías cuantitativas y cualitativas e interpretativas que den cuenta, no sólo de su es- 
tructura, sino de las acciones que emergen entre los distintos actores en los diversos y complejos escenarios en que ocurre la utilización.

El uso de aproximaciones complementarias nos arroja resultados distintos que ayudan a configurar una visión integral de una misma problemática (50). Esto es particularmente importante para los problemas de investigación que se plantean en el ámbito de los sistemas de salud, como es el caso del estudio de la utilización de servicios de salud. Los sistemas de salud están conformados por múltiples instituciones y actores e incorporan procesos de atención y gestión, muy complejos. Resultaría insuficiente abordarlos desde una sola perspectiva de investigación, porque estos procesos están determinados, tanto por la subjetividad y la intersubjetividad de los actores sociales involucrados, por las realidades estructurales de los servicios de salud (recursos físicos, humanos, tecnológicos y financieros), así como por los valores, normas y reglas socialmente construidas en torno a la organización de los servicios de salud.

El uso de la aproximación cualitativa debe verse como una propuesta de aproximación a la realidad social, que permite desentrañar la complejidad de la naturaleza del problema de la utilización de los servicios de salud, ampliando la comprensión de éste fenómeno

\section{REFERENCIAS}

1. Mendoza-Sassi R, Béria JU.Utilización de los servicios de salud: una revisión sistemática sobre los factores relacionados.Cadernos Saude Publica 2001;17(4):819-32.

2. Travassos C, Martins M. A review of concepts in health services access and utilization.Cad. Saúde Pública 2004;20 Sup 2:S190-S198.

3. Frenk J. El concepto y medición de la accesibilidad. Salud Publica Mex 1985;27(5):438-53.

4. Arredondo A, Meléndez V. Modelos explicativos sobre la utilización de servicios de salud: revisión y análisis. Salud Publica Mex 1992;34(1):36-49.

5. Strauss A, Corbin J. Bases de la investigación cualitativa. Técnicas y procedimientos para desarrollar teoría fundamentada. Medellín, Colombia: Editorial Universidad de Antioquia. ; 2002: 11-12.

6. Streubert HJ, Carpentier DR. Qualitative research in nursing. Advancing the humanistic impertative. USA:J.B. Lippincontt Company;1995: 1-14.

7. Gubba EG, Lincoln YS. Paradigmas en competencia en la investigación cualitativa. En: Por los rincones. Antología de métodos cualitativos en la investigación social. Guadalajara: Denman CA, Haro JA, compiladores;2002:113-45.

8. Pathman DE, Fowler-Brown A, Corbie-Smith G. Differences in access to outpatient medical care for black and white adults in the rural south. Med Care 2006; 44(5):429-38.

9. Selden TM, Hudson JL.Access to care and utilization among children estimating the effects of public and private coverage. Med Care 2006;44(5 Suppl):I19-26.

10. Litaker D, Koroukian SM, Love TF. Context and health care access: Looking beyond the individual. Med Care. 2005; 43(6): 531-40. 
11. Niefeld MR, Kasper JD. Access to ambulatory medical and long-term care services among elderly Medicare and Medicaid beneficiaries: organizational, financial, and geographic barriers. Med Care Res Rev. 2005;62(3):300-19.

12. Kirby JB, Kaneda T. Neighborhood socioeconomic disadvantage and access to health care. J Health Soc Behav. 2005; 46(1):15-31.

13. Okoro CA, Strine TW, Young SL, Balluz LS, Mokdad AH.Access to health care among older adults and receipt of preventive services. Results from the Behavioral Risk Factor Surveillance System, 2002. Prev Med 2005;40(3):337-43.

14. Litaker D, Cebul RD.Managed care penetration, insurance status, and access to health care.Med Care. 2003;41(9):1086-95.

15. Larson SL, Fleishman JA. Rural-urban differences in usual source of care and ambulatory service use: Analyses of national data using urban Influence codes. Medi Care.2003;41(7 Suppl): III65-III74

16. Akinci F, Sinay T. Perceived access in a managed care environment: determinants of satisfaction. Health Serv Manage Res. 2003;16(2):85-95.

17. Zhang P, Tao G, Andersen LA. Differences in access to health care services among adults in rural by rural classification categories and age. Aust J Rural Health. 2003;11(2):64-72.

18. Rohrer JE, Kruse G, Borders T, Kupersmith J. Realized access to physician services among the ederly in West Texas. J Rural Health.2003; Winter 19(1):72-8.

19. Andersen RM, Yu H, Wyn R, Davidson PL, Brown ER, Teleki S.Access to edical care for lowincome persons: How do communities make a difference? Med Care Res Rev.2002;59(4):384-411.

20. Almeida RA, Dubay LC, Ko G.Access to care and use of health services by low-income women. Health Care Financ Rev. 2001;Summer 22(4):27-47.

21. Hendryx MS, Ahern MM, Lovrich NP, McCurdy AH. Access to health care and community social capital. Health Serv Res. 2002; 37(1): 87-103.

22. Granados G, Puvvula J, Berman N, Dowling PT.Health care for Latino children: impact of child and parental birthplace on insurance status and access to health services. Am J Public Health.2001;91(11):1806-07.

23. Phillips KA, Mayer ML, Aday LA.Barriers to care among racial/ethnic groups under manager care. Health Aff(Millwood).2000;19(4): 65-75.

24. Flores G, Abreu M, Olivar MA, Kastner B.Access barriers to health care for Latino children. Arch Pediatr Adolesc Med. 1998; 152(11): 1119-25.

25. Weiss E, Haslanger K, Cantor JC. Accessibility of primary care services in safety net clinics in New York City socio-economic and locational determinants of accessibility and utilization of primary health-care. Am J Public Health. 2001; 91(8):1240-45.

26. Coughlin TA, Long SK, Shen YC. Assessing access to care under medicaid: evidence for the nation and thirteen states. Health Aff(Millwood).2005; 24(4):1073-83.

27. Jang M, Lee E, WooK.Income, language, and citizenship status: Factors affecting the health care access and utilization of Chinese Americans.Health Soc Work. 1998;23(2):136-45.

28. Deb P, Li C, Trivedi PK, Zimmer DM.The effect of managed care on use of health care services: Results from two contemporaneous household surveys. Health Economics. 2006;15:743760.

29. Zuvekas AH, Taliaferro GS.Pathways To Access: Health insurance, the health care delivery system, and racial/ethnic disparities, 1996-1999. Health Aff 2003; 22( 2) : 139-53.

30. Fernández-Mayoralas G, Rodríguez V, Rojo E.Health services accessibility among Spanish elderly. Soc Sci Med. 2000;50(1):17-26.

31. Field KS, Briggs DJ. Socio-economic and locational determinants of accessibility and utilization of primary health-care. Health Soc Care Community.2001;9(5):294-308.

32. Redondo-Sendino A, Guallar P, Banegas JR, Rodríguez F, Gender differences in the utilisation of health-care services among the olderadult population of Spain BMC Public Health 2006;16(6):155. 
33. Lester H, Tritter JQ, Sorohan H. Patients' and health professionals' views on primary care for people with serious mental illness: focus group study. BMJ 2005; 330(7500):1122.

34. Noronha KV, Andrade MV. Social inequality in health and the utilization of health services among the elderly in Latin America. Rev Panam Salud Publica. 2005;17(5/6):410-8.

35. Wallace SP, Gutiérrez VF.Equity of access to health care for older adults in four major Latin American. Rev Panan Salud Pública. 2005;17(5-6):394-409.

36. Vicente B, Kohn R, Saldivia S, Rioseco P, Torres S. Patrones de uso de servicios entre adultos con problemas de salud mental en Chile. Rev Panam Salud Publica. 2005;18(4/5):263-70.

37. Buor D.Gender and the utilisation of health services in the Ashanti Region, Ghana.Health Policy 2004;69(3):375-88.

38. Sebata K, Sugisawa Y, Fetters MD, Hiraga A, Ohtaki J, Maezawa M. Focus group investigation on expectations of elderly people from health care facilities and physicians. Nippon Koshu Eisei Zasshi.2002;49(2):114-25.

39. Ngo-Metzger Q, Massagli MP,Clarridge BR, Manocchia M, Davis RB, Lezzoni LI, et al.Linguistic and cultural barriers to care.Perspectives of Chinese and Vietnamese inmigrants. J Gen Inter Med 2003; 18(1):44-52.

40. Hunter JB, de Zapien JG, Denman CA, Moncada E, Papenfuss M, Wallace D, Giuliano AR. Healthcare access and utilization among women 40 and older at he U.S.-Mexico border: Predictor of a routine check-up. J Community Health 2003;28(5):317-33.

41. Berk ML, Schur CL, Chavez LR, Frankel M. Health care use among.undocumented Latino immigrants. Healt Affairs 2000;19(4):51-64.

42. Paim JS, Almeida N. Saúde Coletiva; Uma "nova saúde pública" ou campo aberto a novos paradigmas?. Saúde Pública 1998;34(4):299-336.

43. Almeida N. For a General Theory of Health: preliminary epistemological and anthropological notes. Cad. Saúde Pública 2001;17(4): 753-70.

44. Granda E. ¿A qué llamamos salud colectiva hoy? Rev Cubana Salud Pública.2004;30(2):148-59.

45. Grypdonck MHF.Qualitative Health Research in the Era of Evidence-Based Practice. Qualitative Health Research 2006; 16 (10): 1371-85.

46. Morse JM. Approachs to qualitative-quantitative metodological triangulation. Rev. Nursing Research; 1991:40 (2):120-123.

47. Arias MM. La triangulación metodológica: sus principios, alcances y limitaciones. En: Mercado F, Gestaldo D, Calderón C (comp.).Paradigmas y diseños de la investigación cualitativa en salud. Guadalajara: Universidad de Guadalajara; 2000: 481-99.

48. Morse J, Chung S. Toward holism: the significance of methodological pluralism. International Journal of Qualitative Methods, 2003. Disponible en: www.ualberta.ca/ ijqm. Consultado: febrero 2005.

49. Meetoo D, Temple B. Issues in multi-method research: constructing self-care. International Journal of Qualitative Methods, 2003. Disponible en: www.ualberta.ca/ ijqm Consultado:febrero 2005.

50. Castro R, Bronfman M. Problemas no resueltos en la integración de métodos cualitativos y cuantitativos en la investigación social en salud. En: Castro R, Bronfman M (comp.) Salud y cambio social y política. México: 1999: 49-94. 